\title{
Transfemoral Prostheses Control In a Frame of Intellectual-Synergetic Concept
}

\author{
Aleksandr Poliakov $^{1 *}$, Vladimir Pakhaliuk ${ }^{1}$, Marina Kolesova $^{1}$, Petro Shtanko $^{2}$ and Marina Ovchinnikova ${ }^{3}$ \\ ${ }^{1}$ Sevastopol State University, Sevastopol, Russian Federation \\ ${ }^{2}$ Zaporizhzhya National Technical University, Zaporizhzhya, Ukraine \\ ${ }^{3}$ Humanitarian and Pedagogical Academy, Crimean Federal University named after V.I. Vernadsky, Yalta, Russian Federation \\ ${ }^{*}$ Corresponding author
}

\begin{abstract}
In the control process of transfemoral prostheses in real time, the need to solve a number of non-trivial tasks there arises. The most difficult of them: recognizing the intentions of the disabled person to carry out any movement in the short term and choosing the optimal strategy for moving to the target, taking into account excessive number degrees of freedom of the prosthetic system. At the same time, the optimal should be considered the strategy of movement, which ensures the safety and most comfortable walking of a disabled person from its subjective point of view. Such a strategy can be implemented in a frame of the intellectual-synergistic concept of control. Herewith, the prosthesis control system is designed based on two basic subsystems: the intellectual subsystem - designed to assess the current state and recognize the intentions of the disabled person and the synergetic subsystem - designed to select the optimal strategy for limb movement toward the goal. In this work, the structure of the transfemoral prosthesis control system, realized within the framework of the intellectual-synergistic concept with regard to synergistic quality criteria, is substantiated. It is shown that the use of reasonable synergetic quality criteria allows successfully solving the pseudo-Bernstein problem when planning the movements of controlled transfemoral prostheses and using these movements as reference for the prostheses control system.
\end{abstract}

Keywords-component; transfemoral prosthesis, quality criteria, synergy, Pseudo-Bernstein problem, intent recognition, motion strategy, intellectual-synergetic concept

\section{INTRODUCTION}

The theory of controlling the movements of living organisms, developed by N. Bernstein in the first half of the twentieth century, is now generally recognized, and its main provisions are used not only in the physiology of animals, but also in a number of applied sciences such as cybernetics, automatic control theory, robotics, mechatronics, etc. Particular importance within the theory is the separation of control actions into a number of levels (A - paleo-kinetic regulation of motions, B - synergy, C - spatial field, D - objective or semantic actions; E - symbolic coordinations), each of which controls a certain type of movement [1-4]. N. Bernstein presented the hierarchy of these levels as a result of the development of motor functions in the evolution of a biological object and formulated a hypothesis that, in order to construct movements of varying complexity, commands are given at hierarchically different levels of the Central Nervous System (CNS), and in automating movements this function is transferred to a lower level [2].
Considering the reliability of CNS as a natural biological control system, many artificial analogues with a bio-like hierarchical structure have been developed, which are now widely used in various fields of engineering for the control of complex systems. Their generalized architecture is described in detail, for example, in [5].

In such biotechnical systems as Transfemoral Prostheses (TFP), the most popular are three-level hierarchical systems, including subsystems of higher (HLC), middle (MLC) and lower (LLC) control levels [6]. In most known control systems at the MLC level, the problem of coordinating the movements of TFP elements is solved, as a multi-links system with excessive number degrees of freedom. Its solution can be considered an analogue of self-synchronization of limb muscles, which Bernstein called "muscle synergy" [2].

The question of how and in what sense the CNS solves the problem of coordination of movements under conditions of kinematic and dynamic redundancy of the limb, up to the present time remains the main problem of motor control, which is called the Bernstein Problem (BP) [7]. The same task does not have a unique solution for controlling artificial systems with excessive number degrees of freedom, including TFP. Therefore, as a rule, it is sought to reduce it to a problem with a smaller number of independent variables, by artificially introducing the additional conditions (criteria). In such cases, it is commonly called Pseudo-Bernstein Problem (PBP) [8].

Obviously, the quality of the selected criteria largely determines the quality of the PBP solution when controlling the TFP. From this point of view, special interest is provided by the criteria that make it possible to obtain limb movements with a high level of coordination of movements that are close to biologically natural those. Hypothetically, it can be assumed that such criteria provide coordination of the elements of the artificial system, similar to the muscular synergies of N. Bernstein in the biological system, in connection with which they can be called the Synergistic Quality Criteria (SQC).

In the TFP, as artificial systems with an excessive number of degrees of freedom, the SQC can provide not only the bio-like kinematics of movements, but also the comfort of a disabled person in the process of walking from his subjective point of view. To this end, it is advisable to include Synergetic Subsystems (SS) in the structure of control systems, the algorithms of which are built based on SQC. 
One of the features that distinguish even the most perfect TFP from a natural limb is that it is embedded in the biological system and must perform the functions of an amputated limb. Therefore, in an ideal case, the TFP control system should be a continuation of the CNS and generate motor commands similar to the CNS commands. That is, the TFP control system must "know" the current state of the disabled person, assess it against the set of reference states, be able to learn and determine the most likely tactics and strategy of the disabled person's motor activity in the short term. It should take into account the fact that the possible movements of a healthy person is characterized by a wide variety, including instinctive, reflex, semi-conscious and conscious movements, including inappropriate and suboptimal. The intentions to realize such movements can be peculiar to the disabled, in spite of the fact that they are limited in the possibilities to carry out the whole spectrum of human-specific movements. In such cases, control systems based on information on the environment, the state of the disabled person, his typical movements and reactions to typical stimuli, must generate motor commands aimed at performing the most likely movements that contribute to the realization of the intentions of the disabled person at a given time. In this case, the prediction of intentions is expedient to be performed based on computational algorithms in Intellectual Subsystems (IS).

The presence in the structures of TFP control systems of two synergistically related basic subsystems (SS and IS) makes it possible to increase their reliability and quality by meeting both the objective and subjective needs of the disabled person, provided that it is safe in the process of motor activity. In this case, a complete control system can have a hierarchical structure similar to CNS.

The main purpose of this article is to substantiate the hierarchical structure and mathematical support of TFP control systems, designed based on the Intellectual-Synergistic Concept (ISC).

\section{A VARIANT OF TFP CONTROL SYSTEM ON THE BASIS OF} ISC

\section{A. Synergetic Subsystem}

As is known, despite the kinematic redundancy of most biological objects, including humans, their movements aimed at achieving any goal are predictable in most cases and are characterized by certain constancy. For example, a simple problem of the movement of the hand from point to point is inherent in the regularities that are commonly called kinematic invariants: the profiles of the tangential velocity graphs for different amplitudes and durations have a bell-like shape close to the symmetric [9-12], they generally vary little within and between subjects [13], their variability tends to decrease in practical development of motion [14]. According to S. Engelbrecht, one of the explanations of the observed invariance is that some motions are systematically preferable to others, because they satisfy a certain criterion of quality, which is common to all people [15]. In this regard, they can be considered optimal in relation to this criterion. However, there is no general consensus in the scientific community about what constitutes such a criterion. Nevertheless, we believe that such a criterion exists and is realized by including muscle synergies in the organization of limb movements and, consequently, the movement of biological objects can be called synergistically optimal.

There are many quality criteria for biologically natural movements known as SQC: minimum-jerk [16], minimum energy, minimum torque, minimum time [17], minimum torquechange [18], etc. Including, it has been shown by authors of this article that, for example, the minimum torque-change criterion accurately simulates not only the biologically natural movements of the upper limb [19], but also the lower limb [20]. In addition, we have shown that the SQC can be constructed artificially to realize limb movements with a high synergy index $[21,22]$. Finally, our theoretical studies suggest that the existence of a multitude of experimental data will allow us to find functionals and the corresponding $\mathrm{S}$. $\mathrm{Li}$ and $\mathrm{E}$. Noether symmetries (and hence the corresponding conservation laws) in the future, by verifying inverse variational problems, that support the optimality of synergetic motions [23].

To account for the SQC in the SS algorithms, the Uncontrolled Manifold Concept (UCM) methods can be used [24]. According to the UCM, the configuration space of a control object with an excessive number of degrees of freedom is divided into two subspaces. One of them concerns UCM (UCM subspace) and includes all possible TFP configurations, each of which corresponds to a single set of values of controlled variables from the set of possible ones. The second subspace is orthogonal to the UCM (ORT subspace) and represents the control necessary to achieve the goals of the task [25]. From this point of view, the TFP, as a non-free mechanical system, can be represented as a mathematical model representing a manifold $S$ of dimension $m$, which is described in two ways: by specifying $n$ components of the vector $x=\left(x_{1}(\alpha), \ldots, x_{n}(\alpha)\right)^{T}$ in the form of continuous functions of the $m$-dimensional vector argument $\alpha=\left(\alpha_{1}, \ldots, \alpha_{m}\right)^{T}$ and using the system of $n-m$ equations $\eta_{i}(x)=0, i=1,2, \ldots, n-m[26]$. This allows us to determine the bases $A$ and $K$ of the tangent $T(\alpha)$ and the orthogonal $N(x)$ subspaces of the variety $S$

$$
\begin{gathered}
A=\left(a^{1}, a^{2}, \ldots, a^{m}\right)=\left(\frac{\partial x}{\partial \alpha_{1}}, \ldots, \frac{\partial x}{\partial \alpha_{m}}\right)=\frac{\partial x}{\partial \alpha^{T}}, \\
K^{T}=\left(k^{1}, k^{2}, \ldots, k^{n-m}\right)=\left(\frac{\partial \eta_{1}(x)}{\partial x}, \ldots, \frac{\partial \eta_{n-m}(x)}{\partial x}\right)^{T}=\frac{\partial \eta}{\partial x^{T}},
\end{gathered}
$$

as well as its metric tensors: $G^{\alpha}=A^{T} A$ и $\Gamma^{\eta}=$ $K^{T} K$, respectively. If $\operatorname{rank}(A) \neq m$, then $\operatorname{det}(G)=0$ and the variety $S$ is described incorrectly. That is, in such cases, the components of the vector $\alpha$ are linearly dependent, because their number is greater than the dimension of $T(\alpha)$ and, consequently, the system under study has an excessive number of degrees of freedom.

In order to the condition for the correctness of the description of the manifold $S$ to be fulfilled, one can use some condition that determines the desired motion of the mechanical system. When controlling the TFP, one of the SQCs or a combination thereof can be selected as such a condition.

One of the important advantages of the UCM is the ability to manage the value of the synergy index, which is defined as the ratio of UCM to ORT $[27,28]$. If the UCM component is larger than the ORT component, then the executable variable is controlled by coordinating the motor elements, i.e. with using 
the synergy [28]. Accounting for the SQC in the SS algorithms reduces the synergy index, but this, in turn, leads to improved controllability of the prosthesis and to the realization of bio-like movements.

The SQCs in SS are represented as target functions. For the tests of the TFP control system developed within the framework of the ISC we used the minimum torque change criterion with the coefficients of significance $w_{i}$ (weights) of the active torque $\tau_{i}, i=\overline{1, n}$ for the realization of bio-like motions:

$$
I=\int_{t_{1}}^{t_{2}} \sum_{i}\left(w_{i} \frac{d \tau_{i}}{d t}\right)^{2} \Rightarrow \min
$$

The number of $n$ active torques that is taken into account when implementing criterion (1) is determined by the type of the TFP model. In the experiments, we used the TFP with an artificial controlled polycentric knee joint, controlled magneto rheological dampener (MRD) and an uncontrolled artificial foot [29], the design scheme of which is shown in Figure I.

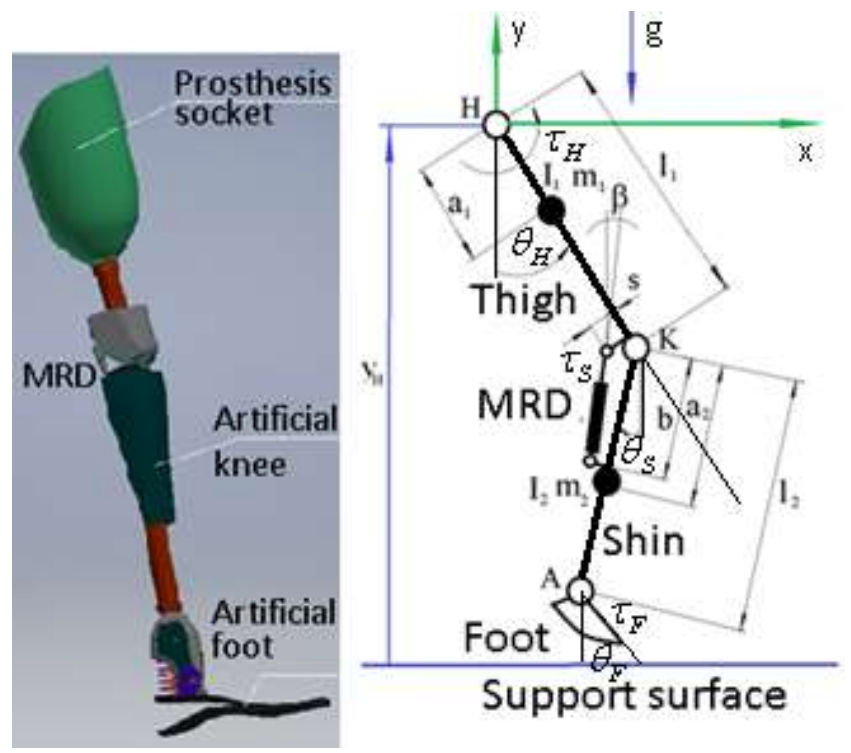

FIGURE I. THE SCHEME OF PROSTHETIC LIMB

The matrix equation of the TFP dynamics in the standard kind has the following form:

$$
D(\theta) \theta+C(\theta, \dot{\theta}) \ddot{\theta}+G(\theta)=B
$$

where $\theta=\left(\begin{array}{lll}\theta_{H} & \theta_{S} & \theta_{F}\end{array}\right)^{T}$ is the vector of rotation angles of the TFP elements (the angle of rotation of the knee joint is determined by the expression: $\left.\theta_{K}=\theta_{H}-\theta_{S}\right) ; D(\theta)$ is a $3 \times 3$ inertial matrix of the TFP; $G(\theta)$ is the vector of the moments, depending on gravitational, damping and inertial forces, determined by the hip joint accelerations; $\mathrm{C}(\theta, \dot{\theta})$ is the vector of moments of centrifugal and Coriolis forces; $B=$ $\left(\begin{array}{lll}\tau_{H} & \tau_{S} & \tau_{F}\end{array}\right)^{T}$ is the vector of the active moments.

Substituting the left side of (2) in (1), and considering that the coordinates of the hip joint $\left(x_{H}, y_{H}\right)$ and the angle of rotation of the femoral component of the prosthetic system $\theta_{H}$ implemented directly by a disabled person, we obtain the system of differential Euler-Poisson equations, the solution of which represents a vector of synergistically optimal solutions $r_{o p t}=$ $\left(\theta_{\text {Kopt }} \theta_{\text {Fopt }} F_{\text {dopt }}\right)^{T}$, where $\theta_{\text {Kopt }}, \theta_{\text {Fopt }}, F_{\text {dopt }}$ are the optimum rotation angles knee, feet and optimum damping force generated in the MRD during the transition TFP from the initial configuration corresponding to the time instant $t_{1}$ to a finite configuration corresponding to the time instant $t_{2}$. The procedure for solving this problem is described in detail in [21].

\section{B. Intellectual Subsystem}

The IS can be considered as an artificial subsystem of the CNS, intended for the realization of physically disabled bio-like movements. That is, it should function like the CNS subsystems, in which the information about typical movements is accumulated and stored, the current state of the environment, the disabled person, his intent to carry out a certain motor action, etc. are estimated. Based on this information, the IS commands are formed in the IS, aimed at transferring the TFP from one state to another. Depending on the type of motor activity of the disabled person, these states can correspond to infinitely close the TFP configurations, as well as substantially different from each other. However, within the framework of the ISC it is assumed that in all cases the transition from one configuration to another is synergistically optimal. In this case, the accounting for the SQC in motion planning algorithms allows solving the PBP and determining pseudo-optimal motions of the TFP elements. II.

The IS scheme in the TFP control system is shown in Figure

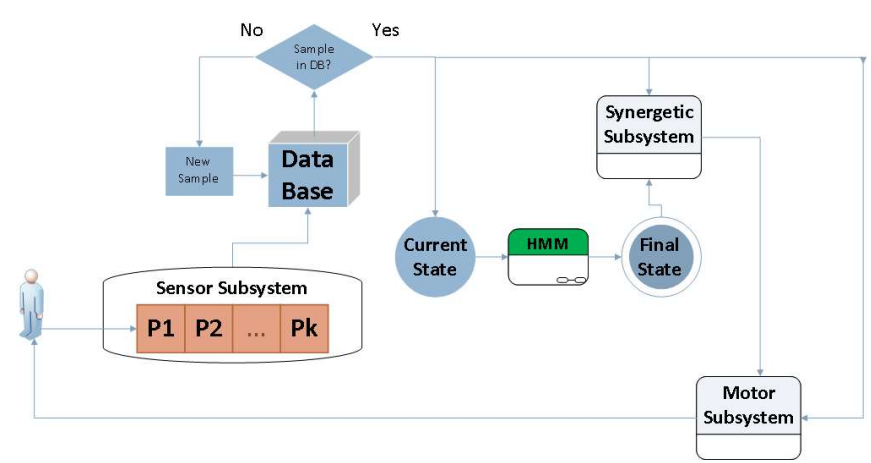

FIGURE II. THE SCHEME OF INTELLECTUAL SUBSYSTEM.

In practice, synergistically optimal movements can be realized only approximately. However, in those cases when the controlled variable is performed with sufficient accuracy, their errors are insignificant. This, in particular, does not contradict the essence of bio-like movements, none of which can be repeated. As you know, this effect, discovered by N. Bernstein, was named by him as "repetition without repetition" (RWR) [1]. However, as noted by M. Latash [30], referring to the principle of abundance, formulated by I. Gelfand and M. Tsetlin [31], the RWR effect indicates that motor redundancy is used by the CNS not to calculate optimal motions, but as some useful device, that requires proper organization and adjustment. Consequently, the IS, like the CNS subsystems, should be able to training, selforganizing and adjustment to ensure the best comfort for the disabled during the walk. Thus, an obligatory element of the IS is an open base of reference patterns of motor activity, which, as the disabled acquire walking skills, is supplemented with new 
data. At the same time, the TFP parameters, as well as the SQC, are adjusted in the learning process based on the experience of the impaired motor activity and its subjective sensations.

Taking into account the fact that the kinematical and dynamic parameters measured with the help of sensors represent only a certain image of the process of impaired motor activity, it is convenient to use the Hidden Markov Models (HMM) to recognize its intentions under given conditions. In this case, the size of the model (the number of states) and the type of observable parameters (discrete or continuous distribution densities of the observed parameters) are of great importance. If the base of reference samples of motor activity is small, then for each motor action, the HMM topology can be described quite simply. The number of states of the model can be taken as equal to the number of informative phases characterizing the reference sample of the motor action cycle, which can be divided into two main phases (stance phase and swing phase) and a number of sub-phases, the quantity of which is relatively small. Each reference model of the motor activity of a disabled person is represented as a sequence of phases (sub-phases) of the realized cycle of movements and a model is constructed for each phase, which includes three states: initial, central and final. The HMM cycle of motor activity is formed by connecting a chain of phase models recorded in a database of reference walk patterns. Thus, the HMM can be considered as a finite automaton consisting of $N$ hidden states $S=\left\{S \_1, S \_2, \ldots, S \_N\right\}$, the transitions between which at each instant $t$ occur according to a certain probability law and are described by the transition probability matrix $A$ [32]. In a certain state $i$, the HMM is characterized by the stationarity of the observed signals over a limited time interval. Upon transition to the new state $j$ at the time $t$, the output parametric vector $x_{t}$ is generated in accordance with the multidimensional probability distribution function $f_{j}(x)$. Thus, the result of the operation of the HMM is the sequence of observations vectors $X=\left\{x_{1}, x_{2}, \ldots, x_{T}\right\}$ of $T$ length.

The process of HMM learning consists in determining the matrix of the probability distribution of the transitions between the states $A$ and the parameters of the weighted Gaussian mixtures $f_{j}(x)=\sum_{i=1}^{M} \omega_{i} p_{i}(x)$,calculated for each state, where $M$ is number of the mixture components; $\omega_{i}$ is the weight of the $i$-th component of the mixture; $p_{i}(x)=$ $\frac{1}{2 \pi^{\frac{D}{2}}\left|\sigma_{i}\right|^{\frac{1}{2}}} \exp \left\{-\frac{1}{2\left(x-\mu_{i}\right)^{2} \sigma_{i}^{-1}\left(x-\mu_{i}\right)}\right\}$ is a normal $D$-dimensional distribution with the vector of estimates of mathematical expectations $\mu_{i}$ and the covariance matrix $\sigma_{i}$.

To recognize the intent of a disabled person using the HMM, the two steps must be taken. The first is to collect a reference data set for calculating the parameters of $\lambda$ model:

$$
\lambda=(A, B, \pi),
$$

where $A=\left\{a_{i j}\right\}_{Q \times Q}, a_{i j}=P\left(X\left(t_{k}\right)=S_{i} \mid X\left(t_{k+1}\right)=S_{j}\right) ; S_{i} \in$ $\left\{S_{i}\right\}_{Q \times 1}, X\left(t_{k+1}\right) \in\left\{X_{i}\right\}_{N \times 1} ; Q$ is a sequence of state models; $N$ is a sequence of hidden state models; $B$ is the probability distribution matrix associated with a set of $Y$ observations of $\mathrm{M}$ size in the state $S_{i}: B=\left\{b_{i j}\right\}_{Q \times N}, b_{i j}=P\left(Y\left(t_{k}\right)=Y_{j} \mid X\left(t_{k}\right)=\right.$ $\left.S_{i}\right) ; \pi$ is the distribution of the vector of initial states: $\pi=$ $\left\{\pi_{i}\right\}_{O \times 1}, \pi_{i}=P\left(X\left(t_{0}\right)=S_{i}\right)$.
Currently, in the learning process of HMM, in the first stage, the two iterative algorithms are commonly used together: Forward-Backward and Baum-Welch [33]. The results obtained are used in the second stage to classify the process based on the Viterbi Algorithm [34] or its modification, called Forward-Only $[35,36]$, which improves the quality of data processing in real time.

The "Forward-Only" algorithm is used to process each signal in order to find the $l$-th state of the probable sequence $l_{t_{k}}$ and the probability associated with each $i$-th state $\gamma_{t_{k}}(i)$. It includes three steps $[35,36]$ :

- Initialization:

$\gamma_{t_{0}}(i)=\pi_{i} b_{i}\left(Y\left(t_{0}\right)\right), 1 \leq i \leq N, l_{t_{0}}=\arg \max \left[\gamma_{t_{0}}(i)\right] ;$

- Recursion:

$$
\gamma_{t_{k}}(i)=\max \left[\gamma_{t_{k}-1}(i) a_{i j}\right] b_{i}\left(Y\left(t_{k}\right)\right) ;
$$

- Probable sequence:

$$
l_{t_{k}}=\arg \max \left[\gamma_{t_{k}}(i)\right] \text {. }
$$

Thus, the "Forward-Only" algorithm allows to compute the most probable sequence of states $L$, including each probable state $l\left(t_{k}\right)$ at the time instant $t_{k}$ and the probability of each $l$-th state $\gamma_{l}\left(t_{k}\right)$.

If we represent a walking cycle with four phases: Flat Foot, Heel Off, Swing, Heel Strike, then each of these phases can be accepted as a hidden state. In this case, the HMM can be represented as a sequence of states shown schematically in Figure III, with the probability distribution matrix of the transitions between states of the form

$$
A=\left\{a_{i j}\right\}=\left(\begin{array}{cccc}
a_{11} & a_{12} & 0.0 & 0.0 \\
0.0 & a_{22} & a_{23} & 0.0 \\
0.0 & 0.0 & a_{33} & a_{34} \\
a_{41} & 0.0 & 0.0 & a_{44}
\end{array}\right)
$$

where $a_{11}+a_{12}=1.0, a_{22}+a_{23}=1.0, a_{33}+a_{34} 1.0, a_{44}+$ $a_{41}=1.0$.

Since the initial state of the model at the time instant $t_{0}$ is not defined, its probability distribution can be chosen arbitrarily, for example, as follows:

$$
\pi\left(t_{0}\right)=\left(\begin{array}{llll}
0.25 & 0.25 & 0.25 & 0.25
\end{array}\right)^{T} .
$$

In the case where one signal is registered $(M=1)$, the result of the HMM operation is a scalar with the distribution law

$$
p_{t_{k}}\left(Y\left(t_{k}\right) \mid \mu\left(t_{k}\right), \sigma\left(t_{k}\right)\right)=\frac{1}{\sigma\left(t_{k}\right) \sqrt{2 \pi}} e^{\left\{\frac{-\left(Y\left(t_{k}\right)-\mu\left(t_{k}\right)\right)^{2}}{2 \sigma\left(t_{k}\right)^{2}}\right\}},
$$

where $t_{k}$ is the current time; $Y\left(t_{k}\right)$ is the observed value; $\mu\left(t_{k}\right)$ is the average value; $\sigma\left(t_{k}\right)$ is the correlation coefficient (for $M>$ 1: $Y\left(t_{k}\right)$ is a vector of observable values of $M \times 1$ size; $\mu\left(t_{k}\right)$ is the vector of average values of $M \times 1$ size, and $\sigma\left(t_{k}\right)$ is the covariance matrix of $M \times M$ size). 


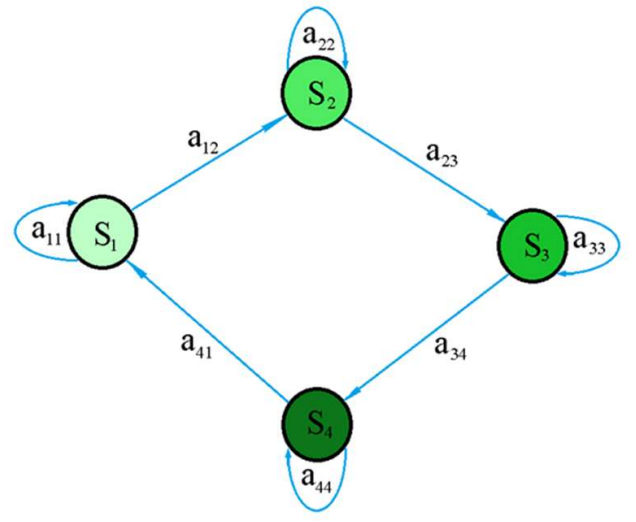

FIGURE III. THE HMM SCHEME CORRESPONDING TO THE MATRIX

(3).

It should be noted that the above algorithm, which makes it possible to determine the most likely intentions of a disabled person during walking, is not optimal and can be modified to minimize recognition time, which requires further theoretical and practical studies in the field of applicability of HMM. In particular, from this point of view, the analysis of Hidden SemiMarkov Models (HSMM), which may prove to be more effective than HMM in solving the intent recognition problem, is of particular interest.

\section{Database of Walking Samples}

To create a database of samples of motor activity, they must be classified. For these purposes, various methods can be used, many of which are described in detail, for example, in a review [37]. However, samples of human activity, such as walking, running, climbing and descending on stairs and others, are very natural in everyday life. Therefore, relatively simple methods can be used for their classification, but they have to differ by high speed. In addition, such methods should provide recognition and classification of movement of a disabled person in real time. If the movement corresponds to one of the samples stored in the database, the TFP control is performed on this pattern until the end of the cycle. Otherwise, such traffic is classified, and information about it is recorded in the database in the form of a new sample. At the same time, the control of the prosthesis up to the end of the movement cycle is carried out on short time intervals, taking into account the actual state of the disabled person at a given time. However, in all cases within the ISC, the movement of the TFP elements at each interval of time is planned taking into account the SQC.

Since each disabled person has anthropometric data characteristic of him and his own behavior, the information in the database of standard walking patterns should take into account these features. But it is impossible to form it for a specific disabled person, due to loss of motor functions. In this regard, as initial information in the database of reference walking samples, we used the results of research available in the literature, processing them in accordance with the principles of similarity of the same type of movements. To assess the similarity of data and the production of reference samples, we used the Linear Fit method, the advantages of which with respect to other methods are justified in $[38,39]$.
When creating a database of reference samples, we took into account the fact that each of the measured motion parameters can be represented as a cyclic function whose graph can be considered a two-dimensional image of the sample parameter. Such a representation allows us to identify the variation of a parameter during a cycle with a two-dimensional object and use well-developed methods for pattern recognition to classify samples, for example, the method of selecting and classifying the contours of objects presenting in the image [40]. The methodological foundations of the process of finding contours are described in sufficient detail in [41].

Analysis of the graphs of changes in the parameters of the motor activity of a person shows that each of them is unique and, in general, characterizes a certain type of activity with its necessary characteristics. Therefore, the contour of the graph can be considered as a characteristic of a sample of motor activity of a certain type. In order to obtain sufficient conditions for the observed activity to belong to a certain class, it is necessary to simultaneously recognize the contours of the graphs of several parameters. Obviously, the quality of solving the problem of recognizing the type of motor activity of a disabled person in this case increases.

We represent the contour of the graph in the form of $N$ points (pixels), numbered from 0 to $N-1$. The point $P_{k}$ with the number $k$ is characterized by the coordinates $P_{k}\left(x_{k}, y_{k}\right)$. Therefore, the contour of the graph can be described by a system of vectors

$$
\left\{\begin{array}{l}
X=\left\{x_{0}, x_{1}, \ldots, x_{N-1}\right\} \\
Y=\left\{y_{0}, y_{1}, \ldots, y_{N-1}\right\}
\end{array}\right.
$$

Applying the Fourier transform with respect to each of the vectors of system (4), we obtain a system of spectral characteristics

$$
\left\{\begin{array}{l}
f_{x}(k)=F(X) \\
f_{y}(k)=F(Y)
\end{array} .\right.
$$

The recognition of the graph outlines represented by the system (5) is based on the method of invariant Xy moments [42] Only central moments of low order $(p+q \leq 3)$ are used here:

$$
m_{p q}=\frac{1}{N} \sum_{k=0}^{N-1}\left(f_{x}(k)-\bar{x}\right)^{p}\left(f_{y}(k)-\bar{y}\right)^{q},
$$

где $\bar{x}=\frac{1}{N} \sum_{k=0}^{N-1} f_{x}(k), \bar{y}=\frac{1}{N} \sum_{k=0}^{N-1} f_{y}(k)-$ medium values.

Moments that are invariant to the operations of rotation, translation, and mirror image for the solution of the problem under consideration are not informative. In addition, it is not necessary to calculate the moments that are invariant to the full group of affine transformations, since It is assumed that the contours of all graphs are viewed from the same point.

If we assume that the accuracy of measuring the coordinates of the graph contour points is within $0<\delta<1 \quad(\delta=1$ corresponds to the highest accuracy), then in the first approximation one can take: $\delta x_{k}=\delta y_{k}$. Then the central moment $\mu_{11}$ can be calculated as follows: 


$$
\begin{array}{r}
\mu_{11}=\frac{1}{N} \sum_{k=0}^{N-1}\left(\delta x_{k}-\delta \bar{x}\right)\left(\delta y_{k}-\delta \bar{y}\right)= \\
\delta^{2} \frac{1}{N} \sum_{k=0}^{N-1}\left(f_{x}(k)-\bar{x}\right)\left(f_{y}(k)-\bar{y}\right)=\delta^{2} m_{11}
\end{array}
$$

In the same way, we get:

$$
\mu_{20}=\delta^{2} m_{20}, \mu_{02}=\delta^{2} m_{02},
$$

and:

$$
\underset{\delta^{3} m_{03} .}{\mu_{12}}=\delta^{3} m_{12}, \mu_{21}=\delta^{3} m_{21}, \mu_{30}=\delta^{3} m_{30}, \mu_{03}=
$$

That is, the sensitivity of the moments (7) and (8) to the change in the accuracy of the measurements is proportional to $\delta^{2}$, and the moments $(9)-\delta^{3}$. Consequently, the process of recognizing the contours of graphs is expedient to produce at least in two stages. In the first (preliminary) stage, a number of reference samples closest to the classified should be selected, using the moments (7) and (8), and in the second stage (refining) - finally choose from the selected series the sample closest to the classified, using the moments (9).

As a measure of the distance between the classified sample and the reference sample, the first stage uses the number

$$
I(A, B)=\sum_{i=11,20,02}\left|\frac{v_{i}(A)-v_{i}(B)}{v_{i}(A)}\right|,
$$

where $\quad v_{i}(A)=\operatorname{sign}\left(h_{i}(A)\right) \cdot \lg \left(h_{i}(A)\right) \quad ; \quad v_{i}(B)=$ $\operatorname{sign}\left(h_{i}(B)\right) \cdot \lg \left(h_{i}(B)\right) ; h_{i}(A), h_{i}(B)$ are Xy moments of the classified (A) and reference sample (B). The measure, allowing to attribute a sample of walking to a certain class, is calculated at the refining stage

$$
I(A, C)=\sum_{i=12,21,30,03}\left|\frac{v_{i}(A)-v_{i}(C)}{v_{i}(A)}\right|,
$$

where $C \subset B$.

As the most informative on the swing phase, the graphs of changes in the angles of rotation of the hip $\theta_{t}$ and knee $\theta_{s}$ joints are chosen, and on the stance phase, a graph of the vertical support reaction $F_{z}$ is added to them. According to the results of the calculation of measures (10) and (11), the observed sample of motor activity is previously referred to one of the reference samples. The final decision on whether the specimen belongs to the reference sample is taken after evaluating its proximity to other informative graphs.

In the event that the observed sample of motor activity is not close to any of the reference samples, it is classified as a new reference sample, characterized by one's own moments of $\mathrm{Hu}$.

Thus, the element of the open (extensible) database of samples of human motor activity is a matrix whose elements are the invariant moments of $\mathrm{Hu}$.

\section{Hierarchical TFP Control System}

The main idea of the ISC is directed to the design of TFP control systems that facilitate the disabled person's realization of the desired motor actions, provided that his safety is ensured. Such control systems can be considered optimal if they provide a comfortable state for the disabled person in the process of walking, i.e. they satisfy the subjective quality criteria that may be different for each disabled person. For example, it is known that various diseases of the locomotor apparatus compel a person to adapt to them, reducing the level of discomfort. Such adaptability can lead to pathological changes in the structure and geometry of individual elements or the skeleton as a whole, up to the development of the state of deformity. But for a sick person, this condition makes it possible to achieve the best level of comfort in these conditions. In this case, obviously, the kinematics and the dynamics of its movements change, which in such cases can be considered "conditionally optimal". In this regard, it can be assumed that the subjective quality criteria do not contradict the objective criteria, such as minimum energy costs, etc. Nevertheless, within the ISC, the maximum quality of comfort that can be achieved by adjusting the TFP parameters In the process of training a disabled person with the skills of performing certain motor actions.

A general scheme of the hierarchical TFP control system that meets ISC requirements is shown in Figure IV.

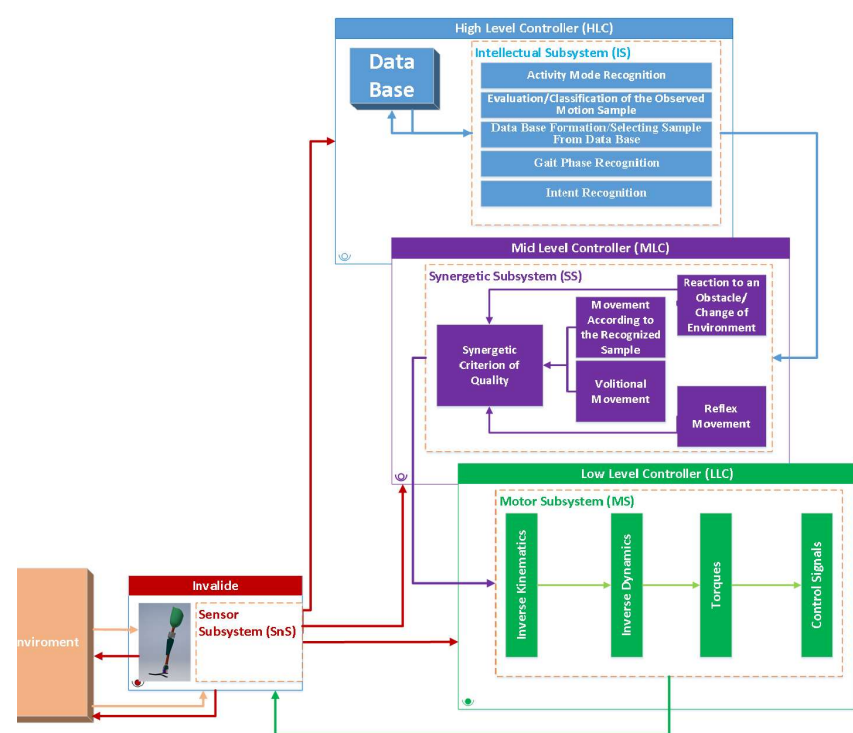

FIGURE IV. GENERALIZED SCHEME OF THE TFP HIERARCHICAL CONTROL SYSTEM.

The RWR effect, formulated above, allows us to state that CNS generates different commands with each new execution of the movement, taking into account the random nature of the variables of the state of the external environment and the person. In this case, according to the theory of sensory corrections, the CNS in real time corrects the commands for the muscles to perform a certain movement based on information received from the peripheral sensory organs about the current state of the person and the results of the movement to the target [2], thereby realizing an adaptive system control with feedback.

A similar strategy is used in the TFP control system. It has a traditional three-level structure, including the HLC, MLC and LLC. At the same time, the IS is used simultaneously in the HLC and MLC algorithms, and the SS is used in the MLC and LLC algorithms. 
In addition to these controllers, a sensor subsystem is used to control movements, which allows to obtain information about the status of the disabled person and the environment in real time and the TFP itself, the regulated parameters of which are: an effort in the bio-like kinematic pair of the artificial knee joint; a variable rigidity of the magnetorheological damper; the stiffness of an artificial ankle joint; the feedback amplifiers of Motor Subsystem (MS). The IS training at the HLC level within the TFP control system is to determine the coefficients of the $A$ matrix in the HMM, which provide the greatest comfort to the disabled person in the performance of the mastered traffic. To ensure the best coordination of movements in the SS, the weighting factors $w_{i}$ in the used SQC are provided.

At the HLC level, the most likely intent of the disabled person is determined, which refers to one of the predefined classes: Reaction on Obstacles/Change of Environment; Reflex Movement; Movement According to the Recognized Sample; Volitional Movement. This classification allows choosing rational tactics of the TFP movement to the target at the level of MLC, the number of time intervals and boundary conditions for the problem of planning a synergistically optimal motion.

For example, sharp flexion of the hip and zero value of the ground reaction force may indicate the realization of a reflex movement by a disabled person, which is performed for a short period of time. In this case, the MLC calculates the boundary conditions, the estimated reflex time, and solves the approximate linear motion planning problem. This takes into account the fact that the reflex movement must be performed quickly, and the accuracy of positioning the TFP elements is less important in this case. If the disabled person wishes, the comfort level can be achieved by performing any possible reflex movement by adjusting the TFP parameters. In this case, it can be represented in the database as a reference movement pattern

As another example, we give an arbitrary (volitional) movement that a disabled person with TFP can implement for a relatively long period of time. As a rule, such movements are not represented in the database and can be performed by a disabled person for the first time. In such cases, the MLC plans synergistically optimal movements over short periods of time. In this case, the boundary conditions at the initial moment of time are determined by the Sensor Subsystem (SnS) information, and at the final moment of time - by attaching virtual increments to them. Despite the fact that the boundary conditions of such a problem are very approximate, the accuracy of its solution in a linear formulation is substantially higher than in the previous example. This is because the linearization of the inverse problems of kinematics and dynamics over short periods of time leads to insignificant errors in the solutions obtained, which, in addition, are compensated for at every step in the solution of the planning problem.

Similarly, the differences inherent in the tactics of planning synergistically optimal movements belonging to other classes can be shown, the number of which, if necessary, can be increased.

At the LLC level, the standard task of controlling the TFP with feedback is solved, which allows implementing the synergetically optimal motions calculated at the MLC level.

\section{RESUlts AND DiscUSSION}

The TFP control system, the structure and software of which were presented above, were tested on the modeling devices detailed in $[43,44]$. In this case, the intentions of the TFP user were set in the form of an agreed sequence of laws for changing the coordinates of the hip joint, the angle of rotation of the femoral component and the speed of the treadmill support surface of the modeling device.

To determine the position and speed of the components of the TFP model in real time, the six IMU sensors with 10 degrees of freedom (two in the thigh, shin and foot component), including three-axis accelerometers LIS331DLH, three-axis gyroscopes L3G4200D, three-axis magnetometers / compasses LIS3MDL and Barometers LPS331AP. The force of the support reaction and the bending moment applied during the stance phase to the artificial foot (Otto Bock, Triton Family) were recorded using pressure and bending sensors FS-L-0095-103ST. The information from the sensors was transmitted to the inputs of the Nucleo-64 STM32F401 controller located inside the bio-like artificial knee joint [29].

The efficiency of the control system was tested on models of standard movements, such as regular walking on a horizontal surface, landing on a chair, rising from a chair, and reflex movements modeled by rapid lifting and lowering of the femoral component during walking. In this case, the samples of standard movements, in contrast to the reflex ones, were recorded in a database of reference samples.

At the originally selected TFP parameters, the IS operation was accompanied by errors, even when modeling the standard movements. Most often this was due to the incorrect definition of the phase of the motion being realized, which led to a discrepancy between the movement of the surface of the treadmill and the TFP model during the swing phase. Such errors, as a rule, led to incorrect contact of the foot with the support surface and loss of stability of the control system. To fix this problem, which has a direct impact on the security of TFP, the database elements have been supplemented with information characterizing the change in the angle of rotation of the knee during walking. In addition, changes were made to the algorithms of the MLC subsystems. In particular, it was decided to plan the initial stage of each type of movement in the SS as unrecognized in the IS. After confirming the compliance of the observed motion with the reference sample, its planning was performed using information about this sample. In all other cases, the Direct Volitional Control (DVC) algorithms were used.

Another problem identified as a result of testing the control system was associated with errors in calculating the control moments and the required stiffness of the magnetorheological damper. We assumed that these errors are primarily determined by the low accuracy of the dynamic model of TFP used, which is further reduced by the linearization. In the general case, this problem could be solved by correctly adjusting the feedback loop gain in the LLC, but the best results were obtained with simultaneous increase in the number of time intervals to which the cycles of movements of all kinds were divided. 
The results of testing the control system showed that for the realization of all types of movements, the greatest reliability is inherent in the DVC algorithms. This fact does not contradict the results of studies carried out earlier by a number of authors. For example, in [45] it was noted that the DVL strategy is effective in controlling the irregular and non-periodic movements, in cases where the location of the foot is decisive for the required movement (walking across rough terrain, climbing / descending stairs, etc.), as well as for non-locomotor actions, such as standing on the spot, positioning the limb in a new position when sitting on a chair, etc. It should be noted that the implementation of DVC algorithms requires significant computational resources, which is the main disadvantage of this strategy in comparison with others.

In general, the results of the testing of the TFP control system developed within the framework of the ISC have shown its effectiveness in controlling the movements of various species that are distinguished by smoothness and high coordination. There is every reason to assume that in real conditions, with proper adjustment of the parameters, the control system will be able to provide the disabled person with the desired level of comfort during walking. The effectiveness of the proposed control system can be significantly improved by using more sophisticated methods of classification and recognition of motor activity or their modification in the IS computational algorithms.

\section{ACKNOWLEDGMENT}

This work has been funded by the Ministry of Education and Science of the Russian Federation in the framework of the base part of State order in the field of scientific activity with the registration No. 115041610028 .

\section{REFERENCES}

[1] N.A. Bernstein, On dexterity and its development. Moscow: Physical Education and Sport, 1991. Н.А. Бернштейн, О ловкости и ее развитии. Москва: Физкультура и спорт, 1991.

[2] N.A. Bernstein, On the construction of motions. Moscow: Book on Demand, 2012. Н.А. Бернштейн, О построении движений. Москва: Книга по Требованию, 2012.

[3] N.A. Bernstein, Essays on the physiology of movements and physiology of activity. Moscow: Medicine, 1966.Н.А. Бернштейн, Очерки по физиологии движений и физиологии активности. Москва: Медицина, 1966.

[4] N.A. Bernstein, The co-ordination and regulation of movements. Elmsford-NY: Pergamon Press, 1967.

[5] L. Wills, S. Kannan, B. Heck, and G. Vachtsevanos, "An open software infrastructure for reconfigurable control systems," Proc. of the 2000 American Control Conf., vol. 4, pp. 2799-2803, 2000.

[6] M.R. Tucker, J. Olivier, A. Pagel, M. Bouri, O. Lambercy, R. Millan, R. Riener, H. Vallery, and R. Gassert, "Control strategies for active lower vol. 12, No 1, pp. 1-29, 2015.

[7] M.L. Latash, M.F. Levin, J.P. Scholz, and G. Schöner, "Motor Control Theories and Their Applications," Medicina (Kaunas), vol. 46, No 6, pp. 382-392, January 2010.

[8] M.L. Latash, Synergy. New York: Oxford University Press, 2008.

[9] W. Abend, E. Bizzi, and P. Morasso, "Human arm trajectory formation," Brain, vol. 105, pp. 331-348, 1982.

[10] J.M. Hollerbach, "Optimum kinematic design for a seven degree of freedom manipulator," In H. Hanafusa, and H. Inoue (Eds.), Robotics research. The $2^{\text {nd }}$ int. symp., Kyoto, Japan, pp. 215-222, Cambridge, MA: MIT Press, 1985.
[11] P. Morasso, “Spatial control of arm movements," Exp. Brain Res., vol. pp. 223-227, 1981.

[12] P. Morasso, “Three dimensional arm trajectories,” Biol. Cyber., vol. 48, pp. 187-194, 1983

[13] R.C. Miall, and P.N. Haggard, "The curvature of human arm movements in the absence of visual experience,” Exp. Brain Res., vol. 103, pp. 421428, 1995.

[14] A.P. Georgopoulos, J.F. Kalaska, and J.T. Massey, "Spatial trajectories and reaction times of aimed movements: Effects of practice, uncertainty, and change in target location," J. of Neurophys., pp. 725-743, 1981.

[15] S.E. Engelbrecht, "Minimum Principles in Motor Control," J. of Math. Psych., vol. 45, pp. 497-542, 2001.

[16] N. Hogan, "An organizing principle for a class of voluntary movements," J. of Neurosc., vol. 4, pp. 2745-2754, 1984.

[17] Y. Uno, M. Kawato, and R. Suzuki, "Formation and control of optimal trajectory in human multijoint arm movement," Biol. Cybern., vol. 61, pp. 89-101. 1989.

[18] M. Kawato, Y. Maeda, Y. Uno, and R. Suzuk,. "Trajectory formation of arm movement by cascade neural network model based on minimum torque-change criterion,” Biol. Cybern., vol. 62, pp. 275-288, 1990.

[19] A. Polyakov, M. Kolesova, and M. Kalinin, "Planning of the manipulator end-effector trajectory by synergetic criterion," Proc. Vol. from the 5-th IFAC workshop DECOM-TT 2007, Cesme-Izmir, Turkey, pp. 185-190, 2007, May 2007.

[20] A. Poliakov, G. Olinichenko, Y. Pashkov, M. Kalinin, V. Kramar, and D. Burkov, "A new design of a unipolar hip endoprosthesis focused on the best quality of life of the patients during the postoperative period," Proc. of the IEEE Int. Conf. on Biomed. Eng. (ICoBE 2012), Penang, Malaysia, pp. 22-27, February 2012.

[21] M. Kolesova, A. Polyakov, and M.Kalinin, "Quantitative and qualitative estimations of the manipulator end-effector trajectory planning by synergetic criterion," Facta Universitatis. Series Mechanics, Automatic Control and Robotics, Vol. 7, No 1, pp. 53-70, 2008.

[22] A. Poliakov, M. Kolesova, P. Bugayov, "Application of hypotheses on the invariant characteristics of the human hand to solve problems of movements planning of anthropomorphic mechanisms," Fundam. and Appl. Prob. of Eng. and Techn., Vol. 5(313), pp. 114-129,2015. A Поляков, М. Колесова, и П. Бугаёв. Приложение гипотез об инвариантных характеристиках руки человека для решения задач планирования движений антропоморфных механизмов," Фундам. и Прик. Проб. Тех. и Технол., Том 5(313), с. 114-129, 2015.

[23] A. Poliakov, M. Kolesova, and P. Shtanko, "Real functions, functionals and conservation laws," Fundam. and Appl. Prob. of Eng. and Techn., in press. А. Поляков, М. Колесова, П. Штанько, “Вещественные функции, функционалы и законы сохранения," ," Фундам. и Прик. Проб. Тех. и Технол., в печати.

[24] G. Schoner, "Recent developments and problems in human movement science and their conceptual implications," Ecol. Psychol., vol. 7, pp. 291314,1995

[25] J.P. Scholz, and G. Schöner, "The uncontrolled manifold concept: identifying control variables for a functional task," Exp. Brain Res., vol. 126, pp.289-306, 1999

[26] V.V. Velichenko, "Matrix-geometric methods in mechanics with applications to the tasks of robotics," Moscow: Science, 1988. B.B. Величенко, "Матрично-геометрические методы в механике с приложениями к задачам робототехники,” Москва: Наука, 1988.

[27] M.L. Latash, J.P. Scholz, and G. Schöner, "Motor control strategies revealed in the structure of motor variability," Exerc. Sport Sci. Rev., vol. 30, No 1, pp. 26-31, 2002.

[28] S. Togo, and H. Imamizu, "Normalized index of synergy for evaluating the coordination of motor commands," PLoS ONE, vol. 10, No 10, pp. 123, 2015.

[29] A. Poliakov, V. Pakhaliuk, N. Lozinskiy, M. Kolesova, P. Bugayov, and P. Shtanko, "Biosimilar artificial knee for transfemoral prostheses and exoskeletons," Facta Universitatis. Ser. Mech. Eng., vol. 14, No 3, pp. 321-328, 2016.

[30] M. Latash, "There is no motor redundancy in human movements. There is motor abundance," Motor Control, vol. 4, pp. 259-261, 2000. 
[31] I.M. Gelfand, and M.L. Tsetlin, "On mathematical modeling of the mechanisms of the central nervous system," In I.M. Gelfand, V.S. Gurfinkel, S.V. Fomin, and M.L. Tsetlin (Eds.), Models of the structuralfinctional organization of certain biological systems, pp. 9-26, Cambridge MA: MIT Press, 1971.

[32] R.J. Elliott, L. Aggoun, and J.B. Moore, Hidden Markov Models. Estimation and Control. New York: Springer Verlag, 1991.

[33] L. Rabineer, "A tutorial on Hidden Markov Models and selected applications in speech recognition," Proc. of the IEEE, vol. 77, pp. 257286, 1989.

[34] A.J. Viterbi, "Error bounds for convolutional codes and an asymptotically optimum decoding algorithm," IEEE Trans. Inform. Theory, vol. IT-13, pp. 260-269, 1967.

[35] N. Abaid, P. Cappa, E. Palermo, M. Petrarca, and M. Porfiri, "Gait detection in children with and without hemiplegia using single-axis wearable gyroscopes," PLoS ONE, vol. 8, No 9, pp. 1-8, 2013.

[36] J. Taborri, S. Rossi, E. Palermo, F. Patane, and P. Cappa, "A novel HMM distributed classifier for the detection of gait phases by means of a wearable inertial sensor network," Sensors, vol. 14, pp. 16223-16234, 2014.

[37] M. Vrigkas, C. Nikou, and I.A. Kakadiaris, "A review of human activity recognition methods," Frontiers in Robotics and AI, vol. 2, Art. 28, pp. 1$28,2015$.

[38] M. Iosa, A. Cereatti, A. Merlo, I. Campanini, S. Paolucci, and A. Cappozzo, "Assessment of waveform similarity in clinical gait data: The linear fit method,” BioMed Res. Int., vol. 2014, Art. ID 214156, 7 pages, 2014.

[39] R. Di Marco, A. Pacilli, E. Scalona, S. Rossi, C. Mazzà, and P. Cappa, "Choosing a similarity index to quantify gait data variability," Gait and Post., vol. 49, Supl. 1, p. S7, 2016.

[40] A.V. Kazbekov, and N.A. Maksimov, "Methods for comparing contours in problems of pattern recognition," Scientific Herald of MSTU, No 185, pp. 37-42, 2012. А.В. Казбеков, Н.А. Максимов, “Методы сравнения контуров в задачах распознавания образов,” Научный вестник МГТУ, №. 185 , C. 37-42, 2012.

[41] J.A. Canny, "A computational approach to edge detection," IEEE Trans. on Patt. Anal. and Mach. Intel., vol. 8, No 6, pp. 679-698, 1986.

[42] M.K. Hu, "Visual pattern recognition by moment invariants," IRE Trans. on Inform. Theory, vol. 8, Issue 2, pp.179-187, 1962.

[43] A. Poliakov, M. Kolesova, P. Bugayov, N. Lozinskiy, Y. Norik, P. Gadkov, and O. Chepeniuk, "System synthesis of a testbench for testing modules and control systems of lower-limb prostheses," Proc. of the 2015 Int. Conf. on Biomed. Eng. and Comp. Technol. (SIBIRCON), Novosibirsk, Russia, pp. 150-155, 28-30 Oct. 2015.

[44] A. Poliakov, P. Gadkov, M. Kolesova, V. Lazarev, P. Bugayov, and P. Shtanko, "System analysis end synthesis of mechatronic testbench for testing modules and control systems of transfemoral prostheses," Proc. of the 8th Biomed. Eng. Int. Conf. (BMEiCON 2015), Pattaya, Tailand, pp. 1-5, 25-27 Nov. 2015.

[45] D.A. Braun, A. Aertsen, D.M. Wolpert, and C. Mehring, "Learning optimal adaptation strategies in unpredictable motor tasks," J. of Neurosc., vol. 29 , No 20 , pp. 6472-6478, 2009. 